\section{Patient-held shared care records for individuals with mental illness}

\author{
Randomised controlled evaluation
}

JAMES P. WARNER, MICHAEL KING, ROBERT BLIZARD, ZARA MCCLENAHAN and SYLVIA TANG

\begin{abstract}
Background Few formalised shared care schemes exist within psychiatry and the evidence base for sharing psychiatric care is weak.
\end{abstract}

Aims To evaluate the utility of patientheld shared care records for individuals with long-term mental illness.

\section{Method Cluster-randomised controlled parallel-group 12-month trial involving 90 patients with long-term mental illness drawn from 28 general practices.}

Results Carrying a shared care record had no significant effect on mental state or satisfaction with psychiatric services. Compared with controls, patients in the shared care group were no more likely to be admitted (relative risk 1.2, 95\% Cl 0.861.67) and attend clinic (relative risk 0.96 , 95\% Cl 0.67-1.36) over the study period. Uptake of the shared care scheme was low by patients and professionals alike.

Subjects with psychotic illness were significantly less likely to use their records (relative risk $0.51,95 \% \mathrm{Cl} 0.27-0.99$ ).

Conclusions Patient-held records may not be helpful for patients with long-term mental illness.

\section{Declaration of interest None.}

Shared care has been defined as "joint participation of GPs and hospital consultants in the planned delivery of care for patients with a chronic condition, informed by an enhanced information exchange over and above routine discharge and referral letters" (Hickman et al, 1994a). Shared care has not been found to improve outcome in other areas of health care, including diabetes (Hayes \& Harries, 1984) and obesity management (Richman et al, 1996). Hickman et al (1994b) described a survey of different models of shared care operating within two health regions. Only 2 of the 65 shared care schemes were for individuals with mental illness. There are few published evaluations of shared care in psychiatry. Essex et al (1990) described a pilot study of patient-held shared care booklets for individuals with schizophrenia based in primary care. Almost none of the local psychiatrists were willing to participate in this pilot, although both general practitioners (GPs) and patients felt that the booklets were useful.

\section{Aims}

The aims of this study were to identify whether carrying a shared care booklet improves mental health, service contact and patient satisfaction and to assess the pattern of use of patient-held records by patients and professionals.

\section{METHOD}

\section{Sample}

Patients aged 16-65 years with long-term mental illness who were registered with a GP and would be receiving follow-up by at least one mental health professional and living in the hospital's catchment area were eligible for recruitment. Long-term mental illness was defined as diagnosis of psychosis, severe non-psychotic disorder, severe personality disorder or other illnesses requiring long-term supervision that resulted in the patient being unable to fulfil at least one of the following: holding down a job; self-care and personal hygiene; performing necessary domestic chores; participating in recreation activities (Kendrick et al, 1995). The disability must be due to one of the following: withdrawal or inactivity, responses to hallucinations, bizarre or embarrassing behaviour, or violence towards self or others.

\section{Design of the shared care record}

The record was designed to be small enough to be convenient, but large enough to take adequate information. The record was A6 size (similar to a European passport) and had three sections. Section 1 (front page) was for recording the patient's initials, date of birth and the names and addresses of GP, psychiatrist, social worker and keyworker. For reasons of confidentiality, the patient's full name and address were not recorded on the record. At the bottom was a return address if the card was found. Section 2 (middle pages) provided space for recording brief clinical notes and medication details (one entry per page). Section 3 , on the back page, was for recording future appointments.

\section{Outcome measures}

The outcome measures chosen were the Behaviour and Symptom Identification Scale (BASIS-32; Eisen et al, 1994), the Brief Psychiatric Rating Scale (BPRS; Overall \& Gorham, 1962), rated 0-6, the Client Satisfaction Questionnaire (CSQ; Larsen et al, 1979), hospital admissions and out-patient clinic attendance and default rates. The BASIS-32 is a self-report questionnaire that provides a comprehensive assessment of psychopathology. Thirty-two items are rated between ' 0 ' for no problem to ' 4 ' for severe problems.

\section{Randomisation}

For the purposes of this study, the unit of randomisation was the patient's general practice. Randomising the patient or individual GP could result in bias, given that the introduction of the patient-held records to some patients or doctors within a practice may result in a change in the culture of care, and this could have an impact on the controls.

A list of all the local general practices in the hospital's catchment area was assembled. 
Practices were numbered sequentially in alphabetical order according to the first line of the address. Practices were randomised to shared care or control status using a computer-generated algorithm.

\section{Recruitment}

We (Z.McC., S.T., J.W) recruited subjects near the time of their discharge (i.e. within a week of the anticipated discharge date as supplied by the ward). Posters were displayed prominently on the wards and the ward staff were reminded regularly about the study. Wards were visited at least weekly during the recruitment periods in order to identify potential subjects. We monitored discharges in order to identify patients who had been discharged without seeing a member of the study team. Attempts were made to see at home any individuals who may have met eligibility criteria but who were discharged before recruitment. If the potential subject met the eligibility criteria and gave written informed consent, the BASIS-32, BPRS and CSQ were completed prior to identifying randomisation status. Subjects in the shared care arm were given a shared care booklet and a written and verbal explanation of how to use it. Control subjects received usual care from the primary care and hospital teams. Apart from the provision of shared care, subjects in both arms were treated equally.

\section{Promulgation to health professionals}

All local GPs, hospital doctors, community nurses and social workers were informed about the shared care study before recruitment began. The study was also presented to GPs, hospital staff and social workers at local meetings. Once a subject had been recruited to the study, a letter was sent to the patient's consultant psychiatrist, GP and other health workers giving information about the recruitment. If the subject was in the intervention arm, further information about the use of the shared care booklet was provided. Brightly coloured stickers identifying the patient as being in the shared care study and prompting the health worker to request the record were attached to the patient's hospital notes and were sent to the GP and other health workers to attach to their records.

\section{Follow-up}

Subjects were assessed at 6 months and 1 year, usually by visiting them at home. These time periods were chosen in order to assess whether there was any attenuation in the impact of shared care over time.

\section{Analysis}

\section{Power and sample size}

The BASIS-32 was used as an outcome measure for the purpose of calculation of the sample size. In studies evaluating the validity and reliability of the BASIS-32 undertaken in the USA (Eisen et al, 1994), the mean total sub-scale score of hospitalised patients was 1.34 (s.d. $=0.68$ ). A difference of 0.7 s.d. in the BASIS-32 score is equivalent to a mean difference in global scores of 0.48 , which is similar to the difference observed between admission and follow-up in the original study by Eisen et al (1994). In order to detect such a difference between the intervention and control groups at the end of the study at a significant level $(\alpha)$ of $5 \%$ (two-sided test) and $80 \%$ power $(1-\beta), 33$ patients needed to be recruited to each arm of the study.

\section{Statistical analysis}

Categorical data were analysed by the $\chi^{2}$ test or comparison of odds ratio (OR) or relative risk (RR) with confidence intervals (CI) where appropriate. Parametric data were analysed using Student's $t$-tests. Longitudinal data were analysed using analysis of covariance. Data were analysed on observed cases (i.e. subjects completing all assessments) and by intention-to-treat using last observation carried forward with all subjects completing the recruitment procedure incorporated into the final analysis. Practice-based analysis was also performed on the main outcome parameters. The intracluster correlation coefficient (ICC) was calculated for baseline values for those practices with more than one recruit using the formula:

$$
\rho_{\text {int }}=\frac{\sigma_{\text {between }}^{2}}{\sigma_{\text {between }}^{2}+\sigma_{\text {within }}^{2}}
$$

where $\rho_{\text {int }}$ is the ICC, $\sigma_{\text {between }}^{2}$ is the between-cluster variance and $\sigma_{\text {within }}^{2}$ is the within-cluster variance (Machin et al, 1997). This can be used to calculate the 'inflation' needed to correct sample size estimates. This is given by the equation:

$$
1+\left(n_{\mathrm{p}}-1\right) \rho_{\mathrm{int}}
$$

where $n_{\mathrm{p}}$ is the mean number of patients per practice.

\section{RESULTS}

\section{Recruitment}

Characteristics of the general practices are shown in Table 1. Of the 252 individuals considered for this study, 90 were recruited (Table 2). Flow through the study and the main reasons for non-recruitment are shown in Fig. 1. There were no significant differences in gender or diagnosis between study entrants and those excluded.

\section{Comparison of shared care and standard care}

There were no significant differences in results of patient-based and practice-based analyses. The results using patient-based data

\begin{tabular}{|c|c|c|c|c|c|c|c|c|}
\hline & \multicolumn{2}{|c|}{ Practices randomised $(n=91)$} & \multicolumn{6}{|c|}{ Practices providing subjects $(n=28)$} \\
\hline & \multirow[t]{2}{*}{ Number of practices } & \multirow[t]{2}{*}{ Number of partners } & \multicolumn{4}{|c|}{$\begin{array}{c}\text { Number of } \\
\text { partners per practice }\end{array}$} & \multirow[t]{2}{*}{$\begin{array}{c}\text { Median (range) partners } \\
\text { per practice }\end{array}$} & \multirow[t]{2}{*}{$\begin{array}{c}\text { Number of practices providing } \\
3 \text { or more subjects }\end{array}$} \\
\hline & & & I & 2 & 3 & $4+$ & & \\
\hline Shared care & 46 & III & I & 5 & 3 & 7 & $5(I-7)$ & 9 \\
\hline Standard care & 45 & 105 & 3 & 5 & 2 & 2 & $4(I-7)$ & 4 \\
\hline
\end{tabular}

Table I Characteristics of randomised practices and those practices from which subjects were recruited 
Table 2 Baseline socio-demographic and clinical characteristics of the sample

\begin{tabular}{lcc}
\hline & Shared care $(n=55)$ & Standard care $(n=35)$ \\
\hline Mean age in years (s.d.) & $36(12.5)$ & 41 (12.6) \\
Male (\%) & $32(56 \%)$ & $16(46 \%)$ \\
Living alone (\%) & $46(84 \%)$ & $29(83 \%)$ \\
Not working (\%) & $47(89 \%)$ & $23(70 \%)$ \\
White (\%) & $49(89 \%)$ & $27(77 \%)$ \\
Diagnosis & & $15(43 \%)$ \\
Schizophrenia (\%) & $23(42 \%)$ & $9(26 \%)$ \\
Depression (\%) & $11(20 \%)$ & $3(9 \%)$ \\
Bipolar disorder (\%) & $8(15 \%)$ & $3(9 \%)$ \\
Personality disorder (\%) & $6(10 \%)$ & $5(14 \%)$ \\
Other (\%) & $7(13 \%)$ & $13(37 \%)$ \\
Baseline assessment & & $1.27(0.86)$ \\
Admission in previous year (\%) & $20(36 \%)$ & $12.5(6.3)$ \\
Mean BASIS-32 (s.d.) & $1.21(0.88)$ & $22.2(4.9)$ \\
Mean BPRS (s.d.) & $12.6(7.0)$ & \\
Mean CSQ (s.d.) & $22.2(4.8)$ & \\
\hline Diagnoses & & \\
\hline
\end{tabular}

I. Diagnoses as recorded in notes.

BASIS, Behaviour and Symptom Identification Scale; BPRS, Brief Psychiatric Rating Scale; CSQ, Client Satisfaction Questionnaire.

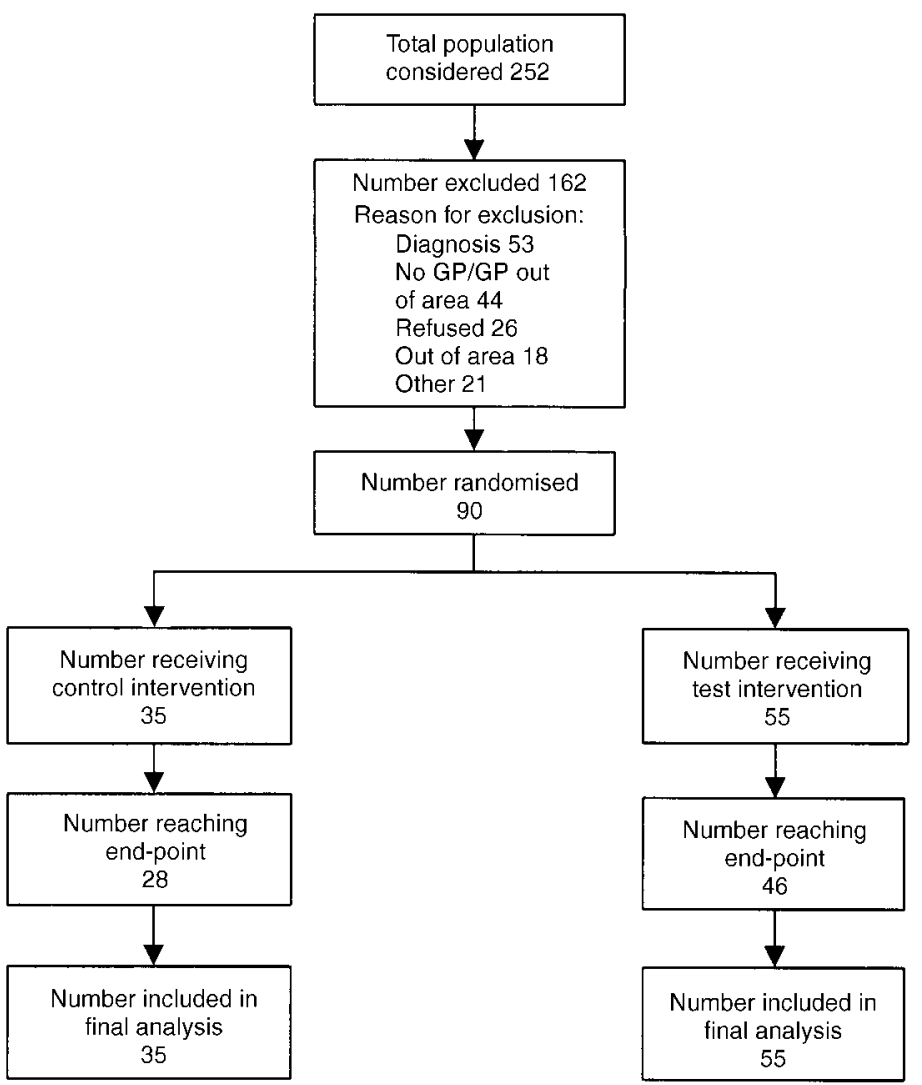

Fig. I Flow diagram of recruitment and attrition.

are presented in Table 3. There were no significant differences in BASIS-32, BPRS or CSQ scores between groups. The number of subjects admitted, in-patient days, number of subjects attending as out-patients and the logistic regression with the following covariates: age, gender, ethnicity, baseline BPRS scores, diagnosis (dichotomised to psychosis/non-psychosis), change of consultant and/or change of GP during the study, admission in the year prior to the study and allocation to shared care or standard care. Two covariates emerged as significant. Admission was more common in patients with psychotic illness $(\mathrm{OR}=5.2,95 \% \mathrm{CI}$ 1.9-14.1) and non-white patients $(\mathrm{OR}=4.1$, 95\% CI 1.2-14.2). Treatment allocation was not a significant predictor of subsequent admissions $(\mathrm{OR}=1.5,95 \%$ CI 0.5-4.0).

Predictors of out-patient attendance and defaults were explored by logistic regression using the following covariates: age, gender, ethnicity, baseline BPRS scores, diagnosis (dichotomised to psychosis/non-psychosis), change of consultant, admission in the year prior to the study and allocation to shared care or standard care. Allocation to shared care did not predict out-patient attendance.

\section{Practice-based analysis}

In order to assess whether there was any cluster effect within practices, betweengroup and within-group differences in demographic variables and baseline satisfaction, BASIS-22 and BPRS scores were compared by one-way analysis of variance. There were no significant differences between practices with respect to patients' gender, age, ethnicity or diagnostic grouping. Between-practice differences in baseline scores were compared by analysis of variance weighted for numbers of subjects per practice. There were significant differences between practices in baseline BASIS32 scores $(F=1.91, \quad$ d.f. $=27$ and 62 , $P=0.02)$ and BPRS scores $(F=1.61$, $P=0.06$ ), but not the number of admissions in the year prior to entry to the study (Kruskal-Wallis $\quad \chi^{2}=28.5, \quad$ d.f. $=27$, $P=0.38$ ). The ICC for baseline values for the BASIS-32 was 0.24 and for the BPRS was 0.30 .

Given that the mean number of patients per practice in this study was 3.2 , the inflation factor for the ICC is 1.53. In other words, given the a priori power calculation based on the BASIS-32-suggested sample of 66 , using the ICC for the BASIS-32 the sample size should be increased to 100 to achieve the same power. 
Table 3 Comparing mean (s.d.) follow-up scores for the Behaviour and Symptom Identification Scale (BASIS-32), Brief Psychiatric Rating Scale (BPRS) and Client Satisfaction Questionnaire (CSQ) between groups using analysis of covariance, with randomisation status as fixed variable and relevant baseline result as covariate'

\begin{tabular}{|c|c|c|c|c|c|c|}
\hline & Shared care $(n=55)$ & Standard care $(n=35)$ & Significance test & $P$ & $\begin{array}{l}\text { Difference } \\
\text { in means }\end{array}$ & $\begin{array}{c}95 \% \mathrm{Cl} \text { for difference } \\
\text { in means }\end{array}$ \\
\hline Mean (s.d.) BASIS-32 & $1.27(0.8 \mathrm{I})$ & $1.20(0.82)$ & $t=0.73$ & 0.38 & 0.06 & -0.28 to 0.41 \\
\hline Mean (s.d.) BPRS & $16.9(9.8)$ & I3.8(8.6) & $t=2.52$ & 0.12 & 3.08 & -0.94 to 7.1 \\
\hline Mean (s.d.) CSQ & $22.3(6.5)$ & $23.4(4.4)$ & $t=0.79$ & 0.37 & -0.89 & -3.38 to 1.59 \\
\hline \multicolumn{7}{|l|}{ Admissions } \\
\hline Number (\%) subjects admitted & $30(55)$ & $15(43)$ & $\chi^{2}=1.17$ & 0.28 & & \\
\hline Median (range) admissions & $0.65(0-7)$ & $0.52(0-5)$ & $z=0.95$ & 0.34 & & \\
\hline Median (range) in-patient days & $7(0-235)$ & $0.71(0-96)$ & $z=0.96$ & 0.33 & & \\
\hline \multicolumn{7}{|l|}{ Out-patient visits } \\
\hline Number (\%) attending clinic & $38(69)$ & $25(71)$ & $\chi^{2}=0.06$ & 0.81 & & \\
\hline Median (range) clinic visits & $1.50(0-17)$ & $\mathrm{I} .46(0-7)$ & $z=0.48$ & 0.63 & & \\
\hline Median (range) clinic defaults & $0.94(0-6)$ & $0.90(0-6)$ & $z=0.27$ & 0.79 & & \\
\hline
\end{tabular}

I. In-patient and out-patient events comparing shared and standard care. Significance test of categorical data by $\chi^{2}$ test. Non-normally distributed continuous data presented as grouped medians (range), significance test with Mann-Whitney U-test.

\section{Use of shared care records}

\section{Subjects' use of records}

During the course of the study no records were returned to the investigator after being lost; 24 (44\%) of the 55 individuals given a shared care record said that they had used it and 12 of these records were retrieved at the end of the study. The number of entries per booklet varied from two to eight (median=4). The number of entries by professionals in these 12 booklets is as follows: hospital doctor $=9, \mathrm{GP}=5$, social worker $=4$, community nurse $=3$.

\section{Professionals' report of record use}

At the end of the study, details of record use were sought from all professionals who had patients allocated to the shared care arm of the study. Thirty-three of the 51 questionnaires $(65 \%)$ were returned. Fourteen professionals reported remembering having seen a record during the study (five GPs, three consultants, two community nurses and four social workers). Eight professionals recalled having records volunteered by the patient and five reported asking the patient for the record. Nine reported writing in a shared care record (three GPs, one consultant, three social workers and two community nurses). Seven of the fourteen respondents who saw a record felt that this was helpful.

\section{Predictors of use of shared care record}

Predictors of record use were explored using logistic regression. Age, marital status, ethnicity, change of consultant or GP, baseline admission rate and baseline scores of the BASIS-32, BPRS and CSQ did not emerge as significant predictors of shared care record use. Diagnosis was a predictor of record use: subjects with psychotic illness were significantly less likely to use their records $(\mathrm{RR}=0.51,95 \%$ CI 0.27-0.99, $P=0.04$ ).

Eighteen $(39 \%)$ of the subjects in the shared care group who were followed up reported losing their shared care record at least once. Ten subjects who reported losing their record at the first follow-up were given a replacement. Nine of this group reported losing the record for a second time. Subjects' reported loss of the record was not significantly associated with diagnosis, gender, change of consultant or GP or admission to hospital over the study period. One of the $13(8 \%)$ non-white subjects in shared care lost their shared care record compared with 17 of the $42(40 \%)$ white subjects $\left(\chi^{2}=4.84, P=0.04\right.$, twotailed Fisher's exact test). Baseline global and sub-scale scores for the BPRS were not related to loss of the record.

\section{DISCUSSION}

Carrying a shared care record did not significantly improve mental health as assessed by the BASIS-32 and BPRS, satisfaction with the services, rates of admission to hospital or out-patient attendance. A large proportion of subjects, mostly patients with schizophrenia and related psychoses, were admitted during the study, reflecting the fact that the subjects recruited had severe mental illness and were vulnerable to relapse.

\section{Shared care in psychiatry}

A variety of shared care models have been suggested (Hickman et al, 1994a), including patient-held records, computer-based shared care, standardised record sheets sent between GPs and hospital, community clinics and liaison. In situations such as psychiatry, where many agencies are often involved in the care of patients, only the first two models are feasible for most patients. Potential advantages to patientheld records include more rapid dissemination of information between professionals, strengthening of the GPs' role and greater involvement of the patient in making decisions about care. However, there are several potential drawbacks, including confidentiality issues, relying on the patient to carry the records and duplication of information.

\section{Methodological issues}

A large number of potential subjects were excluded, although this does not necessarily undermine the validity of the trial. Many potential subjects were excluded because they failed to meet the chosen criterion of 
severe mental illness (Kendrick et al, 1995). Shared care programmes are likely to be of less salience to both patients with less chronic illness and their professional carers. Another principal reason for non-recruitment was that the potential subject or the GP came from outside the catchment area of the hospital. We wanted to test shared care records in an optimal setting with GPs and patients who had contact with the service.

Despite equal numbers of practices being allocated to each treatment arm, more patients were recruited to shared care than to standard care. There are a number of possible explanations for this disparity, one being that there were more GPs $(n=111)$ in the practices allocated to the shared care limb than the standard limb $(n=105)$, but this is unlikely to account for all the difference by itself. Another possible explanation is that several large practices close to the study centre were randomised to the shared care limb and a larger proportion of patients with chronic mental health problems may be registered with these practices because of their proximity to the hospital and the fact that they had general practice partners with an interest in mental health.

\section{Effects of cluster randomisation}

The size of the cluster effect suggests that this study may have been marginally underpowered. When cluster randomisation is used, there may be a difference in the variance between practices and the variance between patients within practices. The variance detected in this study was large (ICC $=0.24$ for the BASIS-32), suggesting that too few subjects were recruited. Nevertheless, there were no trends towards an effect of shared care, and a type II error, although possible, is not likely.

\section{Use of shared care records}

There was a low rate of usage of shared care records among patients and professionals alike, despite the scheme being widely advertised. Patients may have been reluctant to carry shared care records because they were concerned about confidentiality, found the records stigmatising or did not appreciate their potential usefulness. Lack of motivation and organisational abilities in patients with severe mental illness would be another factor to explain the low rate of usage. Professionals may have been reluctant to use the records

\section{CLINICAL IMPLICATIONS}

Patient-held shared care records do not appear to improve mental health or satisfaction with care delivery of patients with long-term mental illness.

Professionals appeared reluctant to use shared care records.

If such schemes are introduced, they should target patients without psychosis.

\section{LIMITATIONS}

- Introduction of shared care records alone does not alter the culture of care delivery.

- Possible inadequate power due to large intraclass correlation coefficient in this sample.

- Service changes during the study may have confounded results.

JAMES P.WARNER, MD, Department of Old Age Psychiatry, Imperial College School of Medicine, London MICHAEL KING, PhD, ROBERT BLIZARD, MSc, ZARA McCLENAHAN, MB, SYLVIATANG, MB, Department of Psychiatry and Behavioural Sciences, Royal Free and University College Medical School, London

Correspondence: Dr James Warner, Department of Old Age Psychiatry, Imperial College School of Medicine, St Charles Hospital, Exmoor St, London WI0 6DZ, UK. Tel: 0208962 4I05; e-mail: j.warner@ic.ac.uk

(First received 20 December 1999, final revision 20 April 2000, accepted 27 April 2000)

because they represent a time-consuming duplication of information, they may have been uncertain about what to write in the records or feared questions or censure about the content. Some professionals may have lacked a feeling of ownership about the study, despite our efforts to involve them.

It is possible that shared care records do not improve patients' well-being no matter how diligently they are used. We cannot assume that better communication will have a positive impact on the outcome parameters measured. Shared care records may enhance communication between professionals but efficacy of communication per se is hard to measure, and all the outcomes chosen are at a level beyond communication and rely on the notion that better communication results in better health or satisfaction or service use.

It is difficult to be dogmatic about the utility of shared care records because of the methodological problems encountered during the study. However, patient-held records (even under the guise of smart cards) should not be introduced to this patient group, at least in the way attempted here, without further evaluation. For a scheme of this nature to work in the future it is likely that patients targeted for shared care will have to be selected carefully, probably excluding those most severely ill patients who may benefit most from shared care. Furthermore, any future shared care scheme should probably be introduced wholesale, with a dedicated coordinator, thus altering the culture of care throughout the service. Paradoxically this would reduce the opportunity to evaluate future schemes under controlled conditions.

\section{REFERENCES}

Eisen, S.V., Dill, D. L. \& Grob, M. C. (1994) Reliability and validity of a brief patient-report instrument for psychiatric outcome evaluation. Hospital and Community Psychiatry, 45, 242-247.

Essex, B., Doig, R. \& Renshaw, J. (1990) Pilot study of records of shared care for people with mental illnesses. British Medical Journal, 300, 1442-1446. 
Hayes, T. M. \& Harries, J. (1984) Randomised controlled trial of routine hospital clinic care versus routine general practice care for type II diabetics. British Medical Journal, 289, 728-730.

Hickman, M., Drummond, N. \& Grimshaw, J. (1994a) A taxonomy of shared care for chronic disease. Journal of Public Health Medicine, 16 447-454.

_ _ _ \& _ (1994b) The operation of shared care for chronic disease. Health Bulletin, 52, 118-126.
Kendrick, T., Burns, T. \& Freeling, P. (1995)

Randomised controlled trial of teaching general practitioners to carry out structured assessments of their long term mentally ill patients. British Medical Journal, 3II, 93-98.

Larsen, D., Attkisson, C. C., Hargreaves, W. A., et al (1979) Assessment of client/patient satisfaction: development of a general scale. Evaluation and Programme Planning, 2, 197-207.
Machin, D., Campbell, M., Fayers, P. M., et al (1997) Sample Size Tables for Clinical Studies (2nd edn). Oxford: Blackwell Science.

Overall, J. E. \& Gorham, D. R. (1962) The Brie Psychiatric Rating Scale. Psychological Reports, I0, 799-812.

Richman, R. M., Webster, P., Salgo, A. R., et al (1996)

A shared care approach in obesity management: the general practitioner and a hospital based service. International Journal of Obesity \& Related Metabolic Disorders, 20, 4I3-4I9. 